\title{
A Two Micron Coherent Differential Absorption Lidar Development
}

\author{
Jirong $\mathrm{Yu}^{* a}$, Mulugeta Petros ${ }^{\mathrm{b}}$, Songsheng Chen ${ }^{\mathrm{a}}$, Yingxin Bai ${ }^{\mathrm{c}}$, Paul J Petzar ${ }^{\mathrm{a}}$, Bo C Trieu ${ }^{\mathrm{a}}$, \\ Grady J Koch ${ }^{\mathrm{a}}$, Jeffrey Y Beyon ${ }^{\mathrm{a}}$, Randal L VanValkenburg ${ }^{\mathrm{a}}$, Michael J. Kavaya ${ }^{\mathrm{a}}$, Upendra N Singh ${ }^{\mathrm{a}}$ \\ ${ }^{a}$ NASA Langley Research Center, MS 468, Hampton, VA USA 23681; \\ ${ }^{\mathrm{b}}$ Science and Technology Corporation, 10 Basil Sawyer Drive, Hampton, VA USA 23666 \\ 'Science System \&Applications, Inc, One Enterprise Parkway, Hampton, VA USA 23666
}

\begin{abstract}
A pulsed, 2- $\mu \mathrm{m}$ coherent Differential Absorption Lidar (DIAL) / Integrated Path Differential Absorption (IPDA) transceiver, developed under the Laser Risk Reduction Program (LRRP) at NASA, is integrated into a fully functional lidar instrument. This instrument measures atmospheric $\mathrm{CO}_{2}$ profiles (by DIAL) from a ground platform. It allows the investigators to pursue subsequent in science-driven deployments, and provides a unique tool for Active Sensing of $\mathrm{CO}_{2}$ Emissions over Night, Days, and Seasons (ASCENDS) validation that was strongly advocated in the recent ASCENDS Workshop.
\end{abstract}

Keywords: Differential Absorption Lidar, Near Infrared Laser,

\section{INTRODUCTION}

NASA Langley Research Center (LaRC) has developed a high energy, pulsed $2 \mu \mathrm{m}$ Differential Absorption Lidar instrument based on coherent heterodyne technique that provides atmospheric $\mathrm{CO}_{2}$ measurements. Since aerosols are abundant in the atmospheric boundary layer, the pulse approach can determine $\mathrm{CO}_{2}$ concentrations as a function of distance with high spatial and temporal resolution, a valuable data product that is not currently available. The short temporal averaging associated with pulsed backscatter also provides high signal-to-noise ratio, enabling high precision measurements. The pulsed lidar approach inherently provides a means for determining range across the scattering targets. The reflected signals can be resolved between aerosols, clouds, and topographical scatters. For column measurements, the pulsed lidar approach can directly eliminate contamination from aerosols and clouds to yield high accuracy measurements. The $\mathrm{CO}_{2}$ absorption lines in the $2.05 \mu \mathrm{m}$ band are ideally suited for $\mathrm{CO}_{2}$ concentration measurements. In particular, the weighting function in the 2- $\mu \mathrm{m}$ region is more favorable for making $\mathrm{CO}_{2}$ measurements near the surface where the sources and sinks of $\mathrm{CO}_{2}$ are located [1]. In spring of this year, the double-pulsed, 2-micron coherent DIAL instrument has been integrated into a mobile trailer, and made the first $\mathrm{CO}_{2}$ concentration measurement. This paper describes the DIAL system configuration and the preliminary measurement results.

\section{DESCRIPTION OF THE DIAL/IPDA HARDWARE}

Figure 1 shows conceptual block diagram of the DIAL/IPDA system. A high-precision and accurate $\mathrm{CO}_{2}$ measurement impose stringent requirements on the lidar transmitter parameters, such as pulse repetition rate, laser power telescope aperture product, laser frequency control accuracy, and the receiver design [2]. The heart of a $\mathrm{CO}_{2}$ DIAL/IPDA system is the transceiver which comprises the laser transmitter, the receiver detector and related optics. The exact wavelengths of the Ho laser are controlled by well defined CW seed laser sources to provide the required on-and-off line wavelength pulses sequentially. The compact, rugged, highly reliable transceiver is based on the unique Ho:Tm:YLF high-energy 2micron pulsed laser technology [3]. The laser architecture is Master Oscillator Power Amplifier (MOPA); both the oscillator and double pass amplifier are side pumped by AlGaAs diode arrays. The temperature controlled rigid optical bench is populated on both sides. The heterodyne receiver and the associated fiber optic couplers, the Faraday isolators to prevent the damage of the seeding lasers, and the laser health diagnostic components are on the back side of the bench while the oscillator and the amplifier are mounted on the front. All the optical mounts are custom designed and have space heritage. They are designed to be adjustable and lockable and hardened to withstand vibrations that can occur in airborne operation. 


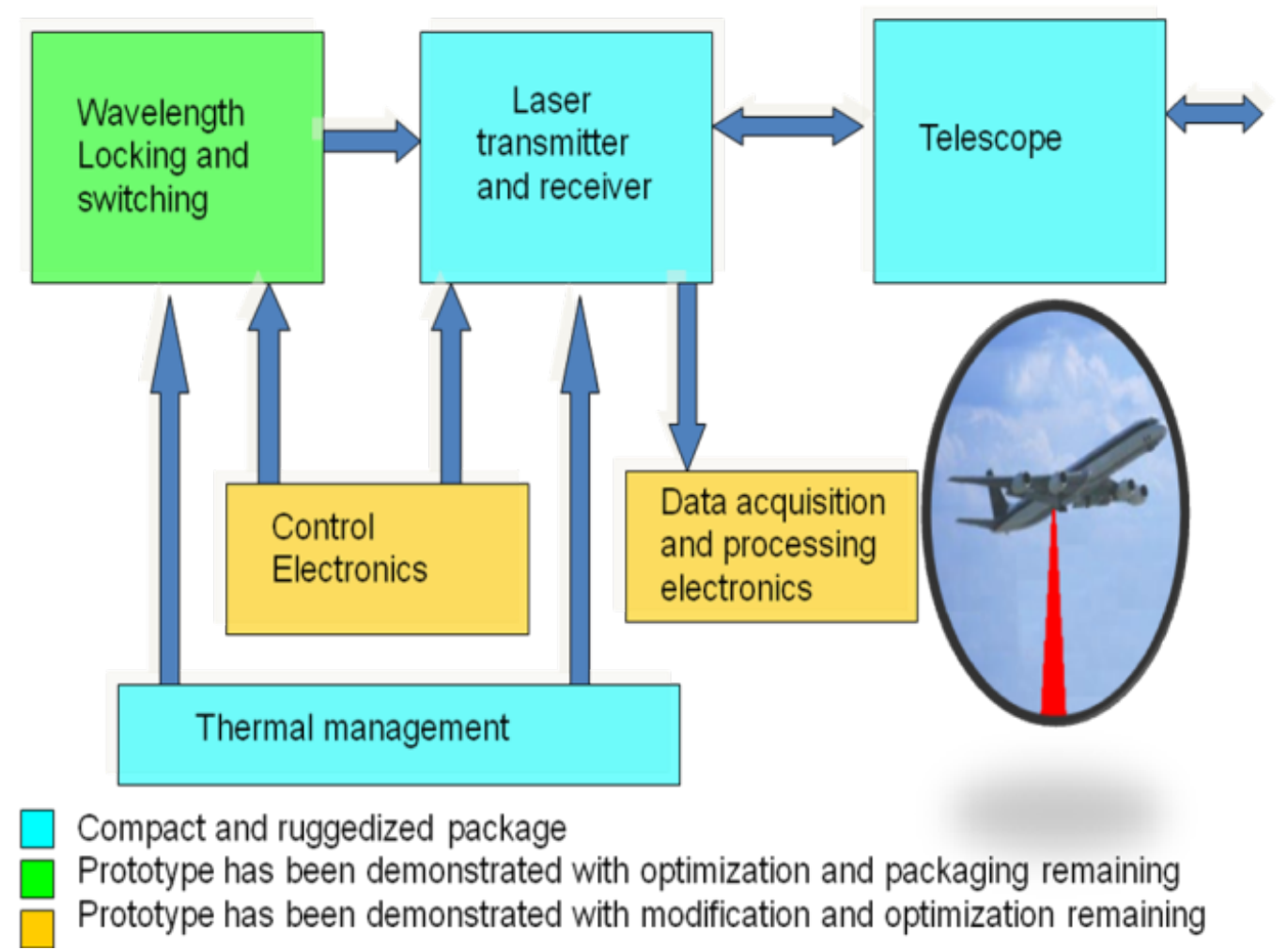

Figure1 $\mathrm{CO}_{2}$ DIAL/IPDA instrument block diagram

The sensitivity and lower detection limit of a DIAL lidar for a specific molecule are defined by the differential absorption cross section between the on- and off-line. We have selected to operate the lidar on the short wavelength wing of $\mathrm{R}(30) \mathrm{CO}_{2}$ line at $2050.967 \mathrm{~nm}\left(4875.749 \mathrm{~cm}^{-1}\right)$ in the side-line operation mode. The side-line position will be optimized for ideal optical depth of 1.0 over the full range of the measurements. The $\mathrm{R}(30)$ line is an excellent absorption line for the measurements of $\mathrm{CO}_{2}$ with regard to the absorption line strength, temperature insensitivity, and lack of interference from other absorption lines [4]. The computations of differential absorption cross-section will take advantage of the most recent full range of spectroscopic measurements including pressure shifts and pressure dependence of line shape [5].

The transmitter is designed to be operated in unique double pulse format to mitigate the effect of the surface reflection difference between the on-and-off line pulses on the precision of the DIAL/IPDA measurement. Figure 2 shows the dynamic characteristic of the Ho upper laser level population in the pumping and lasing period to illustrate the double pulse operation. When the Ho upper laser level population reaches its maximum value, a first Q-switched pulse is generated which extracts the energy stored in the $\mathrm{Ho}^{5} \mathrm{I}_{7}$ upper laser level, resulting in a sharp decrease in the upper laser level population. Then, a new equilibrium between the $\mathrm{Tm}{ }^{3} \mathrm{~F}_{4}$ and $\mathrm{Ho}^{5} \mathrm{I}_{7}$ manifolds is established by a transfer of energy from the excited Tm ions towards Ho ions even though the pump no longer exists. The Ho upper laser level ${ }^{5} \mathrm{I}_{7}$ comes to its second maximum about $150 \mu$ s after the first pulse. The second Q-switch pulse is triggered at this moment resulting again in a sharp drop of the $\mathrm{Ho}^{5} \mathrm{I}_{7}$ population. A unique feature of this laser operation is that it provides two Q-switched pulses with a single pump pulse. The first pulse and the second pulse was injection seeded alternately by the side-line frequency and the off-line frequency. Since the time difference between the pulses is only about $150 \mu \mathrm{s}$, the two pulse foot prints on the ground is greatly overlapped in a flight platform. 


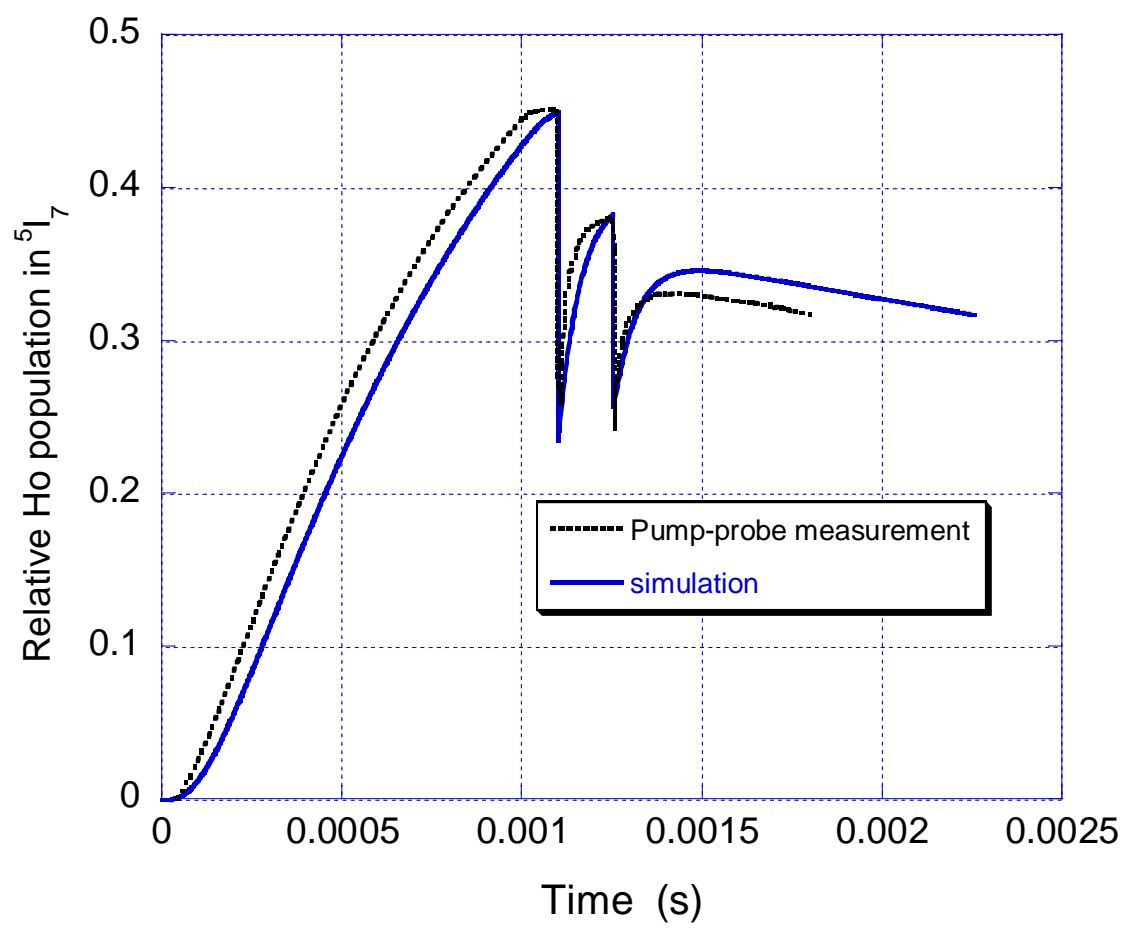

Figure 2 Upper laser level population dynamics during double pulse operation

The heterodyne technique was selected for the receiver design. Heterodyning works by optically mixing the atmospheric backscatter with light from a continuous wave (CW) laser source, called the local oscillator. The local oscillator light is taken from the CW laser which is also injection seeding the pulsed laser. Since the effective receiver bandwidth is very narrow, and the mix with the local oscillator results in an effective amplification of the incoming radiation above the dark noise level of the detector, the heterodyning overcomes the limitations of photo-detectors in the 2- $\mu \mathrm{m}$ wavelength range. The two InGaAs photodiodes in a dual-balanced approach eliminates any amplitude noise from the local oscillator. Extended wavelength InGaAs PIN detectors have been successfully used in the coherent lidar systems at $2 \mu \mathrm{m}$ for many years. A heterodyne instrument does not suffer from spectral impurity of the laser source. Thus, the requirement for the pulse laser transmitter can be relaxed. The transceiver is 11.5 x 26.5 x 6.4 inch $(29$ x $67.3 \times 16.5$ $\mathrm{cm}$ ) in size, and weighted 75lbs.

Tuning and stability of the laser transmitter are critical for making precise and accurate $\mathrm{CO}_{2}$ measurements. To accomplish frequency tuning ability and stability requirements, the technique of injection seeding is used in which the wavelength characteristics of low power, spectrally pure CW lasers are imposed upon the pulsed laser. Three CW lasers, diagrammed in Figure 3, are in an arrangement that gives the level of wavelength control and tuning ability required. The CW lasers are ruggedized versions of commercially available devices originally developed for the NASA Space Readiness Coherent Lidar Experiment (SPARCLE). DIAL requires that the exact wavelength of the laser be known to ensure accurate DIAL results. We have developed a technology for establishing wavelength knowledge to well under $0.05 \mathrm{pm}$ (3.75 MHz). Furthermore, a capability has been added to tune and lock anywhere on the side of the absorption line, so that the amount of absorption to a desired range can be optimized. Tailoring the level of absorption further improves precision and accuracy of the DIAL results.

The means for establishing this unprecedented wavelength stability is to set a master wavelength reference against a sample of $\mathrm{CO}_{2}$ in a gas cell. One of the $\mathrm{CW}$ lasers, called the center-line reference, is passed through the gas cell and actively locked by a frequency modulation spectroscopic technique to hold the laser with respect to absorption line 
center. Tests have shown that long term stabilization over many hours to absorption line center is made to $370 \mathrm{kHz}$ $(0.005 \mathrm{pm})$ standard deviation. A second laser, called the tunable side-line, is referenced to the center-line laser by a heterodyne technique. By monitoring the heterodyne beat signal between the two lasers, the amount of detuning from line center can be determined with high accuracy. An electronic control loop holds the side-line laser at a programmed offset from line center. The side-line laser detuned from the center-line by $4 \mathrm{GHz}$ has been shown to hold the lock to within $322 \mathrm{kHz}(0.004 \mathrm{pm})$ over many hours. The capability of the frequency detuning and locking allows the optimization of the optical depth for measuring atmospheric $\mathrm{CO}_{2}$ concentrations. A third $\mathrm{CW}$ laser provides the off-line wavelength and does not need to be actively controlled because its wavelength is known to be well away from an absorption line. However, it can be locked relative to the reference laser using the same technology as the side line locking if it becomes necessary. The side-line CW laser and off-line CW laser are brought into an optical switch that can be electronically addressed to select the laser. The selected wavelength is then sent to injection seed the pulsed laser alternately in on/off wavelength.

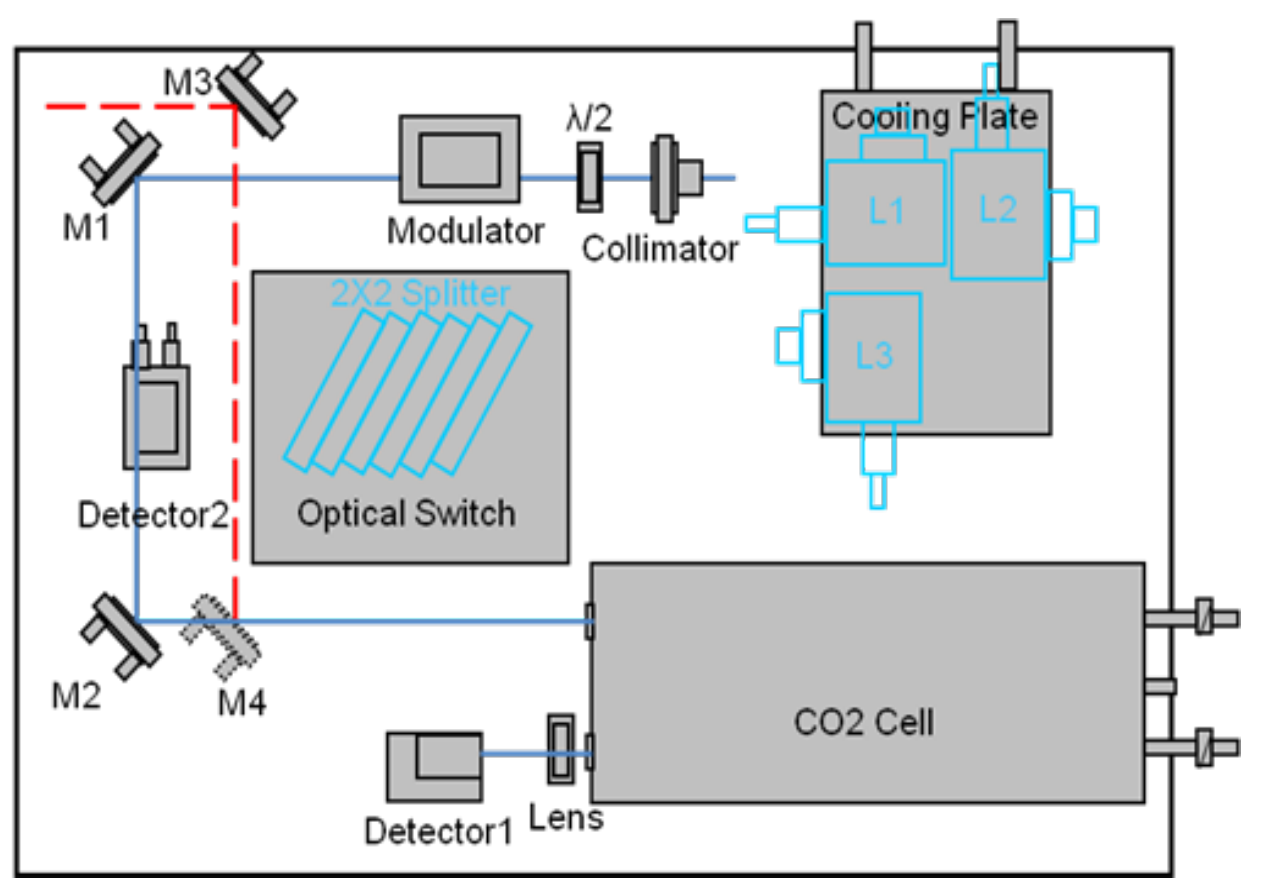

Figure 3 Layout of a prototype wavelength control and switching, L1-L3 are CW lasers used to control wavelengths

The telescope is a custom designed Dall-Kirkham off-axis type with $15 \mathrm{~cm}$ diameter primary mirror size. The secondary mirror was coated with special dielectric coating to reduce the optical loss and to avoid the optical damage due to the high energy of the laser transmitter. The minimum variation in the distance between the primary and the secondary mirrors are maintained by INVAR rod and a custom designed supporting fixture. The focus of the telescope is adjustable, but will be locked for flight.

The data acquisition system is built on two Agilent 10-bit DC252 digitizers housed in a 5 slot Compact PCI 6U crate also manufactured by Agilent. Each digitizer can sample up to $8 \mathrm{GHz}$ with $1 \mathrm{G}$ point acquisition memory. Due to the large acquisition memory size, a long history of data can be monitored before the data extraction process. The two digitizers can be synchronized to the same external trigger signal so that both the trigger and the analog lidar return signals can be digitized simultaneously. The data acquisition software is written in Visual $\mathrm{C}++$ to control the digitization specification such as the sampling frequency, the length of data to be acquired, the trigger signal configuration, the look direction of the telescope, and the specification of data archive. The system runs Microsoft XP with a 64-bit/66 MHz PCI bus, which is capable of transferring data at sustained rates up to $400 \mathrm{MB} / \mathrm{sec}$ to host PC. The system is able to digitize a large amount of lidar return signal at a fast trigger rate with the given specifications. 


\section{DEMONSTRATED PULSED COHERENT $\mathrm{CO}_{2}$ MEASUREMENT PERFORMANCE AND EXPECTED IMPROVEMENT FOR THIS SYSTEM}

During 2007, a coherent DIAL based on pulsed $2 \mu \mathrm{m}$ laser technology demonstrated precise and accurate atmospheric concentration measurement. The laser transmitter is a Q-switched Ho:Tm:LuLiF laser with 90mJ output energy at 5Hz in single pulse format. The R22 of the $\mathrm{CO}_{2}$ absorption line was selected. The on and off line wavelength is controlled by injection seeding the pulse laser by frequency controlled seed lasers. The laser transmitter has been coupled with a coherent heterodyne receiver for measurements of $\mathrm{CO}_{2}$ concentration using aerosol backscatter. Wind and aerosols are also measured with the same lidar and provide useful additional information on atmospheric structure. The DIAL shows excellent performance in accuracy and precision. Measurement of a horizontal column showed a precision of the $\mathrm{CO}_{2}$ concentration to less than $0.7 \%$ standard deviation using a 30 minute (4500 pulse pairs) integration time, and comparison with a co-located in-situ sensor showed the DIAL to measure the same trend of a diurnal variation and to detect shorter time scale $\mathrm{CO}_{2}$ perturbations. The detail of this measurement can be found in reference [6].

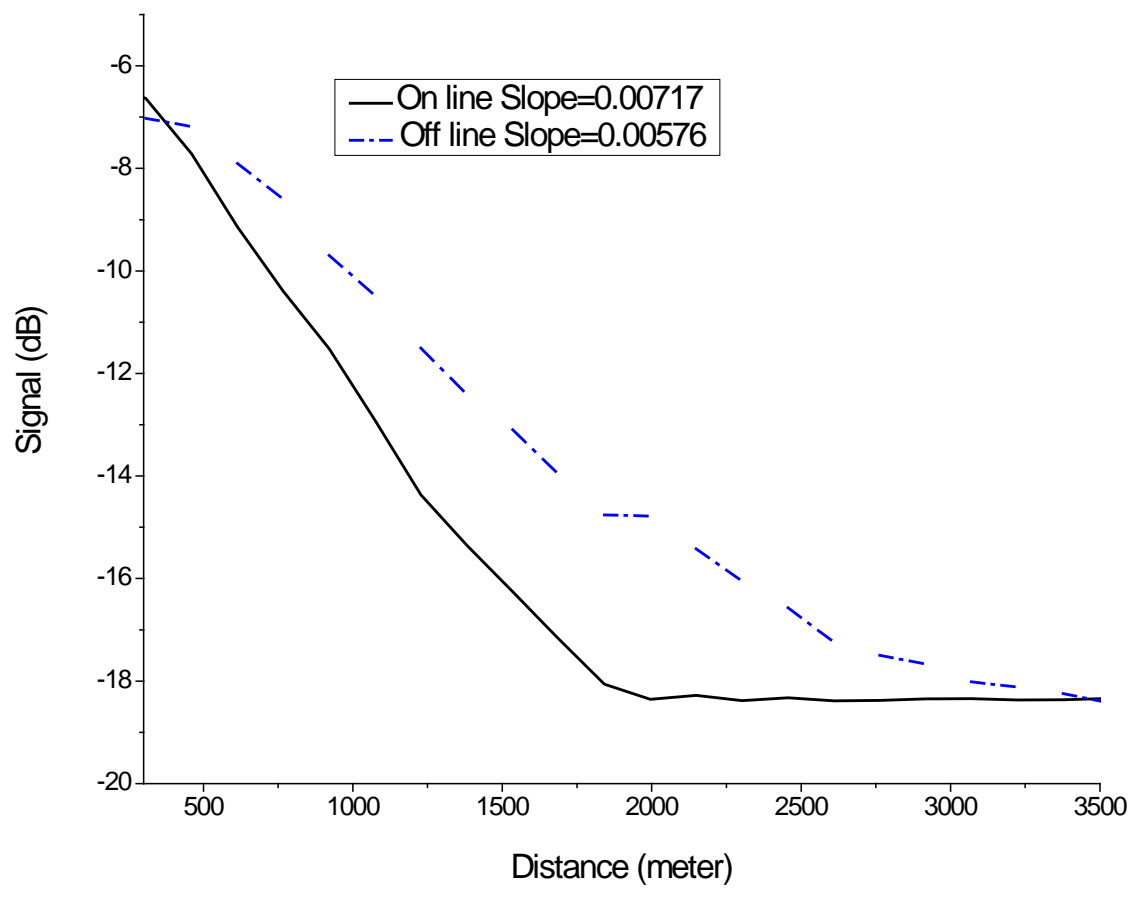

Figure 4. DIAL returning signal strength at on-and-off wavelengths

Figure 4 depicts the returning signal power at on-and-off wavelengths. Total 6000 pairs of pulses are averaged over 10 minutes time period. The slope at on and off line wavelengths is 0.00576 and 0.00717 , respectively. The $\mathrm{CO}_{2}$ concentration calculation from DIAL equation is in progress.

Four significant improvements have been made in this new system compared to the system used in the ground demonstration system described above. First, the proposal system is a $10 \mathrm{~Hz}$, on-and-off pulse pair format pulse transmitter. Not only it improves the measurement precision by mitigating the effect of surface reflection difference between the on-and-off line pulses, but although increases the effective pulse repetition rate by 4 fold. Second, the selection of $\mathrm{CO}_{2}$ absorption line R30 over the R22 further improves the measurement precision due to its negligible water vapor line interference. The parameter of the R30 line is given with good accuracy of $0.4 \%$ for the line strength, 
$0.15 \%$ for the air broadening coefficient, and $0.45 \%$ for its temperature parameter. These accuracies, together with the assumed accuracy of $2.6 \%$ for the pressure shift, are significant to improve the $\mathrm{CO}_{2}$ measurement precision. Third, the system has 8 times higher laser power than the ground demonstration system. It will cover larger measurement distance with increased signal CNR. Fourth, the system is compactly packaged robust system compared to the breadboard system used in 2007 filed measurements.

\section{SUMMARY}

NASA Langley has developed a pulsed, high energy 2-micron lidar transceiver for $\mathrm{CO}_{2}$ concentration measurement by coherent DIAL technique. This technology was demonstrated in a ground based system using a smaller version of breadboard transceiver. The lidar transceiver has now been upgraded; and it is compactly and ruggedly packaged. The upgraded transceiver has 8 times higher optical power and 4 times faster repetition rate than that of the ground based breadboard demonstrated system. It is expected to provide a unique tool for validation of ASCENDS

\section{REFERENCES}

[1] Menzies R. T. and D. M. Tratt, 2003: "Differential laser absorption spectrometry for global profiling of tropospheric carbon dioxide: selection of optimum sounding frequencies for high-precision measurements”, Appl. Opt., 42, pp. 65696577

[2] Ehret G., C. Kiemle, 2005 Requirement definition for future DIAL instruments, final report to ESA contract, p64

[3] Yu J., U. N. Singh, N. P. Barnes, and M. Petros, 1998: “125-mJ diode-pumped injection-seeded Ho:Tm:YLF laser”, Optics Letters 23, 780-782

[4] Regalia-Jarlot, L., V. Zeninari, B. Parvitte, A. Grossel, X. Thomas, P. von der Heyden, and G. Durry 2006: A complete study of the line intensities of four bands of CO2 around 1.6 and $2.0 \mu \mathrm{m}$ : A comparison between Fourier transform and diode laser measurements, J. Quant. Spectr. and Rad. Trans. 101, 325-335

[5] Joly L., Marnas F., Gibert F., Bruneau D., Grouiez B., Flamant P., Durry G., Dumelie N., Parvitte B., and Zeninari V.' 2009: "Laser diode absorption spectroscopy for accurate CO2 line parameters at $2 \mu \mathrm{m}$ : consequences for space-based DIAL measurements and potential biases”, Appl. Opt., 48, 5475-5483

[6] Koch, G. J., J. Y. Beyon, F. Gibert, B. W. Barnes, S. Ismail, M. Petros, P. J. Petzar, J. Yu, E. A. Modlin, K. J. Davis, and U. N. Singh, 2008: "Side-line tunable laser transmitter for Differential Absorption Lidar measurement of $\mathrm{CO}_{2}$ : Design and application to atmospheric measurement”, Appl. Opt. 47, 944-956 